\title{
Magnesium Clofibrate
}

National Cancer Institute

\section{Source}

National Cancer Institute. Magnesium Clofibrate. NCI Thesaurus. Code C66049.

A magnesium salt formulation of clofibrate, an aryloxyisobutyric acid derivate with antihyperlipidemic activity. 